\title{
Correspondence \\ Mathematical Coupling and the True Role of Baseline Severity in Acute Mania Trials
}

\author{
Konstantinos N Fountoulakis*,' and Dimitrios Kontis ${ }^{2}$ \\ 'School of Medicine, 3rd Department of Psychiatry, Aristotle University of Thessaloniki, Thessaloniki, Greece; ${ }^{2}$ Ist Psychiatric Department, \\ Psychiatric Hospital of Attica, Athens, Greece
}

Neuropsychopharmacology (2012) 37, 850; doi: I0.1038/npp.20II.234

Recently two meta-analyses concerning medication in acute mania were published, one of them in Neuropsychopharmacology (Tarr et al, 2011; Yildiz et al, 2011). Both of them report an analysis of change $v s$ initial severity. This kind of analysis has already been reported by a number of meta-analyses of medication in unipolar depression. In one of them, the authors reported a significant relationship between change in raw YMRS score and baseline raw YMRS score for all treatment arms combined (Tarr et al, 2011). However, in the second one, the authors used Hedges' $g$ effect size $v s$ baseline score as percentage of maximum, and reported that higher baseline mania ratings predicted greater improvement with drug but not with placebo (Yildiz et al, 2011). The meta-analytic studies on unipolar depression suggested that medication might not work for mildly depressed patients and hence they should not be prescribed, and as similar findings and conclusions seem to emerge from the meta-analysis of acute mania trials, it seems important to tackle again this issue.

The relation between baseline disease severity and treatment effect has a generic name in the statistical literature, the relation between change and initial value (Blomqvist, 1977), because treatment effect is evaluated by measuring the change of variables from their initial (baseline) values. In psychology, it is also well known as the 'law of initial value' (Jin, 1992).

The use of effect size concept (Cohen's $d$ or Hedges' $g$ ) is more suitable in comparison with raw score as it controls for the variability within studies, and maybe to some extent for the floor and ceiling effects, which every psychometric scale score manifests at very-low or very-high values.

Mathematical coupling occurs when 'one variable directly or indirectly contains the whole or part of another' (Tu and Gilthorpe, 2007). As change scores are the pretreatment scores minus the post-treatment scores, they contain the pretreatment scores. Mathematical coupling can lead to

* Correspondence: Assistant Professor KN Fountoulakis, School of Medicine, 3rd Department of Psychiatry, Aristotle University of Thessaloniki, 6, Odysseos street (Ist Parodos Ampelonon street), 55535 Pylaia, Thessaloniki, Greece, Tel: + 302310 435702, Fax: + 302310 266570,

E-mail: kfount@med.auth.gr an artificially inflated association between initial value and change score, when correlation or regression is used (Tu et al, 2004).

Although there is some concern that group scores are not affected by the mathematical coupling to the extent individual scores are, still the use of standard regression and correlation methods should be considered inappropriate. The use of the effect size $g$ in the $y$ axis by Yildiz et al protected their analysis from the mathematical coupling effect. Thus, their results should be considered closer to reality whereas Tarr's should be considered erroneous.

\section{DISCLOSURE}

Both authors have received support concerning travel and accommodation expenses from various pharmaceutical companies in order to participate in medical congresses. Dr Fountoulakis has also received honoraria for lectures from the Astra-Zeneca, Janssen-Cilag, Eli-Lilly and a research grant from the Pfizer Foundation. Dr Kontis has received honoraria for lecturing by Janssen, Pfizer, and Bristol-Myers Squibb.

\section{REFERENCES}

Blomqvist $N$ (1977). On the relation between change and initial value. J Am Stat Assoc 72: 746-749.

Jin P (1992). Toward a reconceptualization of the law of initial value. Psychol Bull 111: 176-184.

Tarr GP, Herbison P, de la Barra SL, Glue P (2011). Study design and patient characteristics and outcome in acute mania clinical trials. Bipolar Disord 13: 125-132.

Tu YK, Gilthorpe MS (2007). Revisiting the relation between change and initial value: a review and evaluation. Stat Med 26: 443-457.

Tu YK, Maddick IH, Griffiths GS, Gilthorpe MS (2004). Mathematical coupling can undermine the statistical assessment of clinical research: illustration from the treatment of guided tissue regeneration. J Dent 32: 133-142.

Yildiz A, Vieta E, Leucht S, Baldessarini RJ (2011). Efficacy of antimanic treatments: meta-analysis of randomized, controlled trials. Neuropsychopharmacology 36: 375-389. 